\title{
Experimental Measurement of Mixed State Geometric Phase by Quantum Interferometry using NMR
}

\author{
Arindam Ghosh and Anil Kumar \\ NMR Quantum Computation and Quantum Information Group \\ Department of Physics and NMR Research Centre, \\ Indian Institute of Science, Bangalore-560012, India
}

\begin{abstract}
Geometric phase has been proposed as one of the promising methodologies to perform fault tolerant quantum computations. However, since decoherence plays a crucial role in such studies, understanding of mixed state geometric phase has become important. While mixed state geometric phase was first introduced mathematically by Uhlmann, recently Sjöqvist et al. [Phys. Rev. Lett. 85(14), 2845 (2000)] have described the mixed state geometric phase in the context of quantum interference and shown theoretically that the visibility as well as the shift of the interference pattern are functions of geometric phase and the purity of the mixed state. Here we report the first experimental study of the dependence of interference visibility and shift of the interference pattern on the mixed state geometric phase by Nuclear Magnetic Resonance.
\end{abstract}

Key Words : Mixed state geometric phase, Interferometry, NMR.

\section{INTRODUCTION}

When a quantum system undergoes a unitary evolution and comes back to its initial state it acquires a phase. The acquired phase can be of two types; the dynamic phase which depends on the evolution Hamiltonian and the geometric phase, which depends only on the evolution path of the quantum system in the projective Hilbert space [1, 2, 3]. For a two level quantum system $\left(\operatorname{spin}-\frac{1}{2}\right)$, the projective Hilbert space is a sphere and the geometric phase depends on the geodesical solid angle subtended at the center of the sphere by the path of evolution of the state vector. The concept of geometric phase first came in the adiabatic context [2] but later Aharonov et al. [3] gave a non-adiabatic generalization of the theory of geometric phase. In the adiabatic approach the state vector is parallel transported adiabatically to ensure that the system always remains in one of the eigenstates (assuming that the system initially is prepared in one of the eigenstates) of the instantaneous Hamiltonian during the evolution. In the non-adiabatic approach the system is changed abruptly and the system comes back to its initial state through different intermediate 
states. From the total phase acquired by the quantum system, the dynamical phase is eliminated by various methods in order to experimentally measure the geometric phase. In magnetic resonance experiments this is achieved by a spin echo [4]. The theory of geometric phase of a pure quantum system or pure state geometric phase is well understood and has been demonstrated experimentally by various experimental systems such as Nuclear Magnetic Resonance (NMR) [5], single [6] and two photon interferometry [7].

Recently it has been proposed that fault tolerant quantum computation can be performed using geometric phase as it depends on the path and not on the speed of the evolution [8, 9]. To perform computation using geometric phase, it is necessary to understand the relation between geometric phase and decoherence. As decoherence or relaxation leads a system from a pure state to a mixed state, an understanding of the mixed state geometric phase is needed. In 1986 Uhlmann mathematically introduced the concept of mixed state geometric phase [10]. In this paper Uhlmann has taken a large system in pure state and a part or a subsystem in mixed state and pointed out the unitary evolution in which the subsystem is transported in a maximally parallel manner [10]. Recently Sjöqvist et al. have provided a new description for mixed state geometric phase in terms of quantum interferometry [1]]. In a quantum interferometer a quantum system undergoes a series of unitary evolutions, after which the probability of finding the system in one of its eigenstates becomes an oscillatory function of some control parameter. The oscillation pattern of the probability resembles the well known optical interference pattern. According to Sjöqvist et al. the shift of interference pattern is a function of the geometric phase acquired by the quantum system during the unitary evolutions, as well as the purity of the initial internal state (such as, polarization of a photon) of the quantum systems involved in the interferometric operation 11]. The geometric phase can be directly measured from the shift of the interference pattern. Mixed state geometric phase has been experimentally demonstrated by Du et al. [12] using NMR and by Ericsson et al. [13] using single photon interferometry. Du et al. have experimentally demonstrated the mixed state geometric phase by measuring the relative phase change of an auxiliary spin. In the present work we measure the shift of the interference pattern in the Sjöqvist's interferometry model and show that the shift is same as the theoretically predicted geometric phase as a function of mixed state purity. We also demonstrate the effect of mixed state geometric phase on the interference visibility. 


\section{THEORY}

\section{Quantum Interference}

Let us consider the Sjöqvist's interferometry model as shown in Fig [14]. Photons entering the interferometer along a horizontal path are split into two perpendicular paths by a beam splitter $\left(B S_{1}\right)$. On the horizontal path the photons are globally phase shifted, whereas on the other path the internal states of the photons, say the polarization, undergo a unitary evolution $U$. The photons are reflected by two mirrors $\left(M_{1}, M_{2}\right)$ and the two paths meet again at another beam splitter $\left(B S_{2}\right)$. A detector detects the photons coming only along the horizontal path. The detected intensity shows an interference pattern as a function of the phase shift.

As a photon can take one of the two possible paths and in each path it can have one of the two possible polarizations, so the Hilbert space of the combined "path-internal state" system becomes $2^{2} \times 2^{2}$. In NMR, the above interferometry model can be simulated using two coupled spin- $\frac{1}{2}$ nuclei, which have the Hilbert space of identical dimension. One qubit represents the path qubit and the other qubit, termed as spin qubit, represents the internal state.

The equivalent quantum circuit of the Sjöqvist's interferometry model is shown in Fig [2] 14]. The eigenstates $|0\rangle$ and $|1\rangle$ of the path qubit represent the two paths, horizontal and vertical respectively. The path qubit is prepared in the pure state $|0\rangle\langle 0|$ at the beginning of the interferometry operation. The beam splitter is represented by a Hadamard gate given by,

$$
U_{H}=\frac{1}{\sqrt{2}}\left[\begin{array}{rr}
1 & 1 \\
1 & -1
\end{array}\right]
$$

As the phase shifter and the unitary operator ' $U$ ' are path specific, they are represented by two controlled operations, together given by [1]],

$$
U_{C}=\left[\begin{array}{ll}
0 & 0 \\
0 & 1
\end{array}\right] \otimes U+\left[\begin{array}{ll}
e^{i \chi} & 0 \\
0 & 0
\end{array}\right] \otimes \mathbb{1} .
$$

Depending upon the state of the first qubit, the operator $U_{C}$ either applies $U$ on the second qubit or phase shifts the first qubit. The mirrors in Fig 1 represent NOT gates, given by,

$$
U_{M}=\left[\begin{array}{ll}
0 & 1 \\
1 & 0
\end{array}\right]
$$


The input state of the combined "path-spin" system can be written as [14],

$$
\rho_{i n}=(|0\rangle\langle 0|)^{P} \otimes \rho_{0}^{S}
$$

where $\mathrm{P}$ stands for path and $\rho_{0}^{S}$ is the density matrix corresponding to the initial state of the spin qubit which can be either pure or mixed. The initial density matrix $\rho_{i n}$ is transformed into the final density matrix $\rho_{\text {out }}$ as,

$$
\rho_{\text {out }}=U_{H} U_{M} U_{C} U_{H} \rho_{\text {in }} U_{H}^{\dagger} U_{C}^{\dagger} U_{M}^{\dagger} U_{H}^{\dagger}
$$

Substituting the matrix forms of the operators given by Eq.(11,3) we obtain [1],

$$
\rho_{\text {out }}=\frac{1}{4}\left[\left(\begin{array}{ll}
1 & 1 \\
1 & 1
\end{array}\right) \otimes U \rho_{0}^{S} U^{\dagger}+\left(\begin{array}{rr}
1 & -1 \\
-1 & 1
\end{array}\right) \otimes \rho_{0}^{S}+e^{i \chi}\left(\begin{array}{rr}
1 & 1 \\
-1 & -1
\end{array}\right) \otimes \rho_{0}^{S} U^{\dagger}+e^{-i \chi}\left(\begin{array}{ll}
1 & -1 \\
1 & -1
\end{array}\right) \otimes U \rho_{0}^{S}\right] .
$$

The detected signal in the horizontal path $(|0\rangle$ eigenstate of path qubit) is given by the trace of the reduced density matrix of the spin qubit corresponding to the $|0\rangle$ state of the path qubit. The output intensity is given by [14],

$$
\begin{aligned}
I & =\frac{1}{4} \operatorname{Tr}_{S}\left(U \rho_{0}^{S} U^{\dagger}+\rho_{0}^{S}+e^{-i \chi} U \rho_{0}^{S}+e^{i \chi} \rho_{0}^{S} U^{\dagger}\right) \\
& =\frac{1}{2}\left(1+\left|\operatorname{Tr}_{S}\left(U \rho_{0}^{S}\right)\right| \cos \left[\chi-\arg \operatorname{Tr}_{S}\left(U \rho_{0}^{S}\right)\right]\right) \\
& =\frac{1}{2}(1+\nu \cos [\chi-\phi]),
\end{aligned}
$$

where the amplitude of oscillation $\nu=\left|\operatorname{Tr}_{S}\left(U \rho_{0}^{S}\right)\right|$ is called the visibility of interference and the shift $\phi=\arg \operatorname{Tr}_{S}\left(U \rho_{0}^{S}\right)$ depends on the unitary operator ' $U$ ' acting on the spin qubit density matrix $\rho_{0}^{S}$.

A mixed state can be thought of a mixture of several pure states incoherently weighted by their respective probabilities. Therefore, the interference pattern given by Eq[7] takes the following form for a mixed input spin state[11],

$$
I=\sum_{k} w_{k} I_{k}=\frac{1}{2}\left(1+\sum_{k} w_{k} \nu_{k} \cos \left[\chi-\phi_{k}\right]\right)
$$

where the index $\mathrm{k}$ denotes individual pure states with probabilities $w_{k}$. The above equation can be written in the form of Eq[7 as

$$
I \propto 1+\tilde{\nu} \cos (\chi-\tilde{\phi}),
$$

by defining mixed state phase shift $\tilde{\phi}$ and visibility $\tilde{\nu}$ as [11],

$$
\tilde{\phi}=\arg \left(\sum_{k} w_{k} \nu_{k} e^{i \phi_{k}}\right)
$$




$$
\tilde{\nu}=\left|\sum_{k} w_{k} \nu_{k} e^{i \phi_{k}}\right|
$$

\section{Geometric phase and parallel transport condition}

The parallel transport condition for any state vector $|\psi(t)\rangle$ is given by,

$$
\langle\psi(t) \mid \dot{\psi}(t)\rangle=0
$$

which means that the phase does not change when $|\psi(t)\rangle$ evolves to $|\psi(t+\delta t)\rangle$ for infinitesimal $\delta t$. When a mixed state given by the density matrix $\rho_{m}(t)$ evolves under a unitary operator $A(t)$, the condition given in Eq[12 leads to [11],

$$
\operatorname{Tr}\left[\rho_{m}(t) \dot{A}(t) A^{\dagger}(t)\right]=0 .
$$

This condition although necessary is not sufficient to determine the unitary operator $A(t)$ as it can determine $A(t)$ only up to $\mathrm{N}$ phase factors, where $\mathrm{N}$ is the dimension of the Hilbert space. The $\mathrm{N}$ phase factors can be determined from the conditions [15],

$$
\left\langle k(t)\left|\dot{A}(t) A^{\dagger}(t)\right| k(t)\right\rangle=0, \quad k=1,2,3 \ldots \ldots, N,
$$

where $|k(t)\rangle$ are the orthonormal eigenstates of $\rho_{m}(t)$. The unitary operator $A(t)$, obtained by solving the above conditions, parallel transports the mixed state density matrix $\rho_{m}$ so that the dynamical phase becomes identically zero. The geometric phase $\gamma_{g}$, acquired by a mixed state when the state evolves under $A(t)$ along a curve $\Gamma$, is given by [11],

$$
\gamma_{g}[\Gamma]=\arg \operatorname{Tr}\left[\rho_{m} A(t)\right]=\arg \left(\sum_{k} w_{k} \nu_{k} e^{i \beta_{k}}\right),
$$

where $e^{i \beta_{k}}$ is the geometric phase associated with the $k^{t h}$ pure state. The expression for the geometric phase given by Eq[15] is similar to the expression for the interferometric phase shift given by Eq10 and therefore the interferometric phase shift directly gives the geometric phase of the spin qubit.

In the present work we consider the mixed state of a spin- $\frac{1}{2}$ particle. The density operator of a spin- $\frac{1}{2}$ particle can be in general written as,

$$
\rho_{m}=\frac{1}{2}(1+\vec{r} . \vec{\sigma})
$$


where the length 'r' of the Bloch vector $\vec{r}$, is equal to one for pure states, less than one for mixed states and remains unchanged during unitary evolution of the state. The components of $\vec{\sigma}$ are the Pauli matrices, $\vec{\sigma}=\left[\sigma_{x}, \sigma_{y}, \sigma_{z}\right] . \rho_{m}$ represents a mixture of two of its eigenvectors with eigenvalues $\frac{1}{2}(1 \pm r)$.

Let us consider that the Bloch vector $\vec{r}$ for a mixed state $(\mathrm{r}<1)$ traces out a cyclic curve in the Bloch sphere which subtends a geodesically closed solid angle of $\Omega$. During the process the two eigenstates of the density operator with eigenvalues $\frac{1}{2}(1 \pm r)$ acquire geometric phase $\mp \frac{\Omega}{2}$ respectively[16]. The quantity $\sum_{k} w_{k} \nu_{k} e^{i \phi_{k}}$ (Eq10 and [1]), using the fact that $\nu_{k}=1$ for cyclic evolution[1]], becomes,

$$
\begin{aligned}
\sum_{k} w_{k} \nu_{k} e^{i \phi_{k}} & =\frac{1}{2}(1-r) e^{i \frac{\Omega}{2}}+\frac{1}{2}(1+r) e^{-i \frac{\Omega}{2}} \\
& =\cos \left(\frac{\Omega}{2}\right)-i r \sin \left(\frac{\Omega}{2}\right) .
\end{aligned}
$$

Using the expression given by Eq17 the shift of interference pattern (Eq10) and the visibility (Eq 11) for mixed state can be respectively written as,

$$
\tilde{\phi}=-\arctan \left(r \tan \left(\frac{\Omega}{2}\right)\right),
$$

and

$$
\tilde{\nu}=\sqrt{\cos ^{2}\left(\frac{\Omega}{2}\right)+r^{2} \sin ^{2}\left(\frac{\Omega}{2}\right)} .
$$

In the present paper, we have experimentally measured the above frequency shift and the visibility using a two qubit system, by NMR. The frequency shift directly gives the geometric phase of the spin qubit.

\section{EXPERIMENTAL PROCEDURE}

Experiments were performed on Carbon-13 enriched ${ }^{13} \mathrm{CHCl}_{3}$ dissolved in $\mathrm{CDCl}_{3}$. The ${ }^{13} \mathrm{C}$ and ${ }^{1} \mathrm{H}$ nuclei form a two qubit system with a J-coupling of $209 \mathrm{~Hz} .{ }^{1} \mathrm{H}$ and ${ }^{13} \mathrm{C}$ respectively are used as the path and spin qubits. The spin-lattice $\left(T_{1}\right)$ relaxation times of ${ }^{13} \mathrm{C}$ and ${ }^{1} \mathrm{H}$ at room temperature were measured as $21 \mathrm{~s}$ and $16 \mathrm{~s}$ respectively, and the spin-spin $\left(T_{2}\right)$ relaxation times were measured to be $0.29 \mathrm{~s}$ and $3.4 \mathrm{~s}$ respectively. All the experiments were performed using a Bruker DRX $500 \mathrm{MHz}$ (11.2 Tesla) NMR spectrometer where the resonance frequencies for ${ }^{13} \mathrm{C}$ and ${ }^{1} \mathrm{H}$ are $125.76 \mathrm{MHz}$ and $500.13 \mathrm{MHz}$ respectively. The pulse programme is given in Fig 3 . The pulse programme contains several parts which are described below: 


\section{Creation of Pseudo-pure state (PPS)}

The "path-spin" system is first prepared in a pseudo-pure state using the method of spatial averaging [17]. The pulse sequence is as follows,

$$
\left(\frac{\pi}{3}\right)_{x}^{H}-G_{z}-\left(\frac{\pi}{4}\right)_{x}^{H}-\frac{1}{4 J_{C H}}-(\pi)_{y}^{H, C}-\frac{1}{4 J_{C H}}-\left(\frac{\pi}{4}\right)_{\bar{y}}^{H}-(\pi)_{\bar{y}}^{H, C}-G_{z},
$$

where the superscript $\mathrm{H}$ or $\mathrm{C}$ identifies the spin (proton or carbon respectively) on which the r.f. pulse is applied and the subscript $x$ or $y$ determines the phase of the pulse. $J_{C H}$ is the J-coupling and $G_{z}$ indicates a $z$-gradient which destroys all coherences ( $x$ and $y$ magnetizations) and retains only longitudinal magnetization ( $z$ magnetization component). At the end of this sequence the system is prepared in the $|00\rangle$ pseudo pure statei [17].

\section{Creation of Mixed state}

After preparing the $|00\rangle$ PPS, an $\alpha$ degree pulse is applied on the carbon spin followed by a $z$ gradient. In the Bloch representation it creates a mixed state vector whose length $\mathrm{r}=\cos \alpha[r<1$ for $0^{\circ}<\alpha \leq 90^{\circ}$, where the value of $\mathrm{r}$ determines the purity of the state. The above pulse programme can be written as,

$$
(\alpha)_{x}^{C}-G z
$$

\section{The Interferometer}

The Hadamard gate (Beam Splitter) is implemented by the sequence $\left(\frac{\pi}{2}\right)_{y}^{H}-(\pi)_{x}^{H}[18]$. The important operation of the interferometry part is the controlled operation $U_{C}$. $U_{C}$ contains a controlled phase shift gate applied on the path qubit and a controlled $U$ operation acting on the spin qubit.

\section{Controlled Phase shift:}

The controlled phase shift gate in the present context is different from the conventional two-qubit gate. Here both the controlling and the target qubits are the path qubit. The path qubit is phase shifted by $\chi$ when it is in the $|0\rangle$ (horizontal path) state. The output of the phase shift gate $U_{\chi}$ can be written as,

$$
U_{\chi}|00\rangle=e^{i \chi}|00\rangle ; U_{\chi}|01\rangle=e^{i \chi}|01\rangle ; U_{\chi}|10\rangle=|10\rangle ; U_{\chi}|11\rangle=|11\rangle \text {. }
$$

The pulse sequence for $U_{\chi}$ is [18],

$$
\left(\frac{\pi}{2}\right)^{H}-(\chi)_{\bar{y}}^{H}-\left(\frac{\pi}{2}\right)^{H}
$$




\section{Controlled $U$ :}

In the present case $U$ is a geometric phase shift operator. The controlled geometric phase shift operator is implemented by evolving the spin qubit in a cyclic path (the 'Slice Circuit') in the Bloch sphere as shown in Fig 4 , when the path qubit is in state $|1\rangle$ (vertical path). This is achieved by pulsing only on the $|10\rangle-|11\rangle$ subsystem. Two transition selective $\pi$ pulses are applied on the $|10\rangle-|11\rangle$ transition of ${ }^{13} C$ with phases differing by $(\pi+\phi)$ [19]. They cause the Bloch vector to flip along one path, $\Gamma_{1}$ and come back to its initial orientation along a different path, $\Gamma_{2}$. The loop $\left(\Gamma_{1}, \Gamma_{2}\right)$ subtends a geodesically closed solid angle of $\Omega=2 \phi[\underline{5}]$. A spin echo sequence has been incorporated to eliminate the dynamical phase. The pulse sequence is given by,

$$
\tau-(\pi)_{x}^{H}-(\pi)_{\theta}^{|10\rangle-|11\rangle}-(\pi)_{\theta+\pi+\phi}^{|10\rangle-|11\rangle}-(\pi)_{\bar{x}}^{H},
$$

where $\tau$ is the total time of the two transition selective pulses. The second $(\pi)_{\bar{x}}^{H}$ pulse in the above sequence restores the sign of the ${ }^{1} H$ magnetization.

The mirror (NOT gate) is implemented by a $(\pi)_{x}^{H}$ pulse. It converts the state $|0\rangle$ (horizontal

path) to state $|1\rangle$ (vertical path) and vice-versa. The sequence $\left(\frac{\pi}{2}\right)_{y}^{H}-(\pi)_{x}^{H}$ for the Hadamard gate is repeated after the mirror, in order to implement the second beam splitter $\left(B S_{2}\right)$.

\section{Measurement}

At the end of the interferometric operations, both the qubits were detected after applying a $z$ gradient and a reading $\pi / 2$ pulse on the detection qubit. The diagonal part of the density matrix was then tomographed using the line intensities normalized to the respective equilibrium spectra [20].

\section{RESULTS}

The intensity of the signal detected only in the horizontal path is proportional to the total population of the $|00\rangle$ and $|01\rangle$ levels as these two energy levels correspond to the $|0\rangle$ state (horizontal path) of the path qubit. For each $\chi$ the final density matrix (diagonal part only) was tomographed and the sum of the $|00\rangle$ and $|01\rangle$ populations was plotted as the intensity. Data were collected at 37 equidistant values of $\chi$ ranging from $-360^{\circ}$ to $360^{\circ}$ to obtain the full interference pattern. Fig [5] shows the ${ }^{1} H$ and ${ }^{13} C$ spectra for different $\chi$ values for pure initial state of the spin qubit. The three low intensity lines in the carbon spectra arise from the natural abundant carbon coupled to 
deuterium in the solvent $\mathrm{CDCl}_{3}$. The intensities (sum of $|00\rangle$ and $|01\rangle$ populations) calculated from the normalized spectral line intensities are plotted as a function of phase shift $\chi$. Fig 5 (a) shows the pattern when $U$ (the geometric phase shift operator) was not applied. As expected no shift in the pattern from $\chi=0$ was observed. Whereas Fig $\left[5\right.$ (b) shows the pattern corresponding to $\Omega=180^{\circ}$. A shift of $-90^{\circ}$ was observed as expected according to Eq 18 for pure state $(r=1)$. In each plot the solid line represents the expected theoretical curve.

The 'shift - geometric phase' relationship (Eq[18)

For mixed input state of the spin qubit, the pattern shifts from $\chi=0$ for non-zero geometric phase. The amount of shift is a function of both the purity of the input state as well as the geometric phase of the spin qubit. Fig [ shows the dependence of interferometric shift on the geometric phase and the purity of mixed state for $\Omega=60^{\circ}$ (6, a), $\Omega=90^{\circ}$ (6, b) and $\Omega=120^{\circ}$ (6) c). For a particular value of $\Omega$ and r, experiments have been performed for ten equidistant points of $\chi$ in the range $\left[-90^{\circ}, 0^{\circ}\right]$. The data were fitted with a function $\mathscr{F}(\nu, \phi)=\nu \cos (\chi-\phi)$, to calculate the shift. The shift is zero

for $\mathrm{r}=0$ and the shift is $-\frac{\Omega}{2}$ for $\mathrm{r}=1$ in all the three cases. The solid line in each plot represents the theoretical curve. Spectra corresponding to $\alpha=0^{\circ}, 30^{\circ}, 50^{\circ}, 70^{\circ}$ and $90^{\circ}$ have been shown for $\chi$ $=30^{\circ}$ (6. $\mathrm{a}$ ), $\chi=40^{\circ}$ (6. $\mathrm{b}$ ) and $\chi=60^{\circ}$ (6. $\mathrm{c}$ ).

The 'visibility - geometric phase' relationship (Eq 19)

The visibility of interference or the amplitude of oscillation is given by the difference between the maximum and the minimum intensities in the interference pattern. The visibility was measured for different purity ' $r$ ' of the spin qubit state. Fig [7 shows the visibility as a function of ' $\mathrm{r}$ ' for $\Omega=120^{\circ}$ (77) a), $\Omega=180^{\circ}$ (77, b) and $\Omega=360^{\circ}$ (77 c). $\Omega=360^{\circ}$ makes the visibility independent of $\mathrm{r}$, while for $\Omega$ $=180^{\circ}$ the visibility changes linearly with $\mathrm{r}$. The experimental data matches the expected behavior (solid line) given by Eq[19. Spectra corresponding to $\alpha=0^{\circ}, 30^{\circ}, 60^{\circ}$ and $90^{\circ}$ (for $\Omega=180^{\circ}, \alpha=89^{\circ}$ was applied instead of $90^{\circ}$ as the shift according to Eq 18 becomes undefined for $\Omega=180^{\circ}$ and $\mathrm{r}=$ 0 ) have been shown adjacent to each plot. While recording the spectra the value of $\chi$ was chosen according to the shift of pattern given by Eq18. All the experiments for $\Omega=360^{\circ}$ was performed at $\chi=0^{\circ}$ and for $\Omega=180^{\circ}$, at $\chi=-90^{\circ}$. For $\Omega=120^{\circ}, \chi$ was chosen same as the shift predicted by $\mathrm{Eq} 18$ 


\section{CONCLUSION}

The study of mixed state geometric phase has become important ever since geometric phase was proposed as a possible method of performing fault tolerant quantum computing. The pure state geometric phase is well understood and well studied by various experimental methods. Here we have reported the first experimental measurement of mixed state geometric phase directly from the shift of a quantum interference pattern. We have experimentally measured the visibility and the shift of the interference pattern as a function of the purity of the input mixed state which agree with the theoretically expected results. This study shows that NMR interferometry is one of the possible experimental methods to measure geometric phase of a pure as well as a mixed state. Future

directions include studies of non-cyclic geometric phase [21] and applications of geometric phase in fault tolerant quantum computations.

\section{Acknowledgments}

We gratefully acknowledge Prof. K. V. Ramanathan for discussions. The use of DRX-500 high resolution liquid state spectrometer of the NMR Research Centre, Indian Institute of Science, Bangalore, funded by Department of Science and Technology (DST), New Delhi, is gratefully acknowledged. AK acknowledges "DAE-BRNS" for "Senior Scientist scheme", and DST for a research grant. 
[1] S. Pancharatnam, Proc. Indian Acd. Sci. A 44 (1956) 247.

[2] M. V. Berry, Proc. R. Soc. London. Ser. A 392 (1984) 45.

[3] Y. Aharonov, J. S. Anandan, Phys. Rev. Lett. 58 (1987) 1593.

[4] R.R. Ersnt, G. Bodenhausen, A. Wokaun, Principles of Nuclear Magnetic Resonance in One and Two Dimensions, Clarendon press, Oxford, 1987.

[5] D. Suter, K. T. Mueller, A. Pines, Phys. Rev. Lett. 60 (1988) 1218.

[6] P. G. Kwiat, R. Y. Chiao, Phys. Rev. Lett. 66 (1991) 588.

[7] J. Brendel, W. Dultz, W. Martienssen, Phys. Rev. A. 52 (1995) 2551.

[8] J. A. Jones, V. Vedral, A. Ekert, G. Castagnoli, Nature 403 (2000) 869.

[9] Shi Liang Zhu, Z. D. Wang, Phys. Rev. Lett. 91 (2003) 187902.

[10] A. Uhlmann, Rep. Math. Phys. 24 (1986) 229.

[11] Eric Sjöqvist, A. K. Pati, A. Ekert, J. S. Anandan, M. Ericsson, D. K. L. Oi, V. Vedral, Phys. Rev. Lett. 85 (2000) 2845.

[12] J. Du, P. Zou, M. Shi, L. C. Kwek, J. Pan, C. H. Oh, A. Ekert, D. K. L. Oi, M. Ericsson, Phys. Rev. Lett. 91(10) (2003) 100403.

[13] M. Ericsson, D. Achilles, J. T. Barreiro, D. Branning, N. A. Peters, P. G. Kwiat, Phys. Rev. Lett. 94 (2005) 050401.

[14] A. Hosoya, A. Carlini, S. Okano, quant-ph/0405116

[15] A. G. Wagh, V. C. Rakhecha, Phys. Lett. A 197 (1995) 107.

[16] J. S. Anandan, Phys. Lett. A 129 (1988) 201.

[17] D.G. Cory, M. D. Price, T.F. Havel, Physica D 120 (1998) 82.

[18] J. A. Jones, R. H. Hansen and M. Mosca, Jl. of Mag. Res. 135 (1998) 353.

[19] Ranabir Das, "Developments in Quantum Information Processing by Nuclear Magnetic Resonance", Ph.D. Thesis, Indian Institute of Science (2004).

[20] Ranabir Das, T. S. Mahesh, Anil Kumar, Chem. Phys. Lett. 369 (2003) 8.

[21] Eric Sjöqvist, Phys. Lett. A 286 (2001) 4. 


\section{FIGURE CAPTIONS}

Figure 1. The Sjöqvist's interferometry model [14]. $\mathrm{BS}_{1}$ and $\mathrm{BS}_{2}$ are two beam splitters which split a photon beam in two perpendicular paths. $\mathrm{M}_{1}$ and $\mathrm{M}_{2}$ are two mirrors. The unitary operation ' $U$ ' acts on the internal state of the photons traveling along the vertical path while the photons traveling along the horizontal path are phase shifted by $\chi$ by the phase shifter. The detector detects the photons coming along the horizontal path only.

Figure 2. The quantum equivalent circuit of Sjöqvist's interferometry model using two qubits [14]. One qubit corresponds the path while the other corresponds the spin (internal state). The single qubit gates used are Hadamard $\left(U_{H}, \mathrm{Eq} \underline{\mathbb{1}}\right)$ and $\operatorname{NOT}\left(U_{M}, \mathrm{Eq} \underline{3}\right)$. The control operation $U_{C}$ consists of a control phase shift gate acting on the first qubit and a control ' $U$ ' operator acting on the second qubit (Eq,2).

Figure 3. The pulse programme to perform NMR interferometry. The black and empty boxes represent $\pi / 2$ and $\pi$ pulses respectively while the gray boxes represent pulses with flip angle given on the top. The phase of a pulse is given at top of the pulse. The gray Gaussian shaped pulses are transition selective soft $\pi$ pulses applied on the $|10\rangle-|11\rangle$ Carbon transition. $\mathrm{G}_{Z}$ is the $z$-Gradient pulses applied to kill all transverse magnetizations. $\mathrm{J}$ is the coupling between ${ }^{1} H$ and ${ }^{13} \mathrm{C}$ and is equal to $209 \mathrm{~Hz}$ in the present case. The pulse programme can be divided in four major parts, namely the PPS, preparation of mixed state, interferometer and the measurement, which are discussed in detail in the text. The last $\pi / 2$ pulses (shown by broken lines) on each spin is the detection pulse and is applied at the detected spin one at a time.

Figure 4. The slice circuit for cyclic evolution of a state vector [for pure state $|\vec{r}|=1$ and for mixed states $|\vec{r}|<1$ ] in the Bloch sphere [19]. In the present case, the $|10\rangle-|11\rangle$ subsystem is transported through a closed loop using two transition selective $\pi$ pulses applied at phases $(\theta)$ and $(\theta+\pi+\phi)$ on the $|10\rangle-|11\rangle$ Carbon transition. The first $\pi$ pulse takes the second qubit from state $|0\rangle$ to state $|1\rangle$ along the path $\Gamma_{1}$, while the second $\pi$ pulse brings the state back from $|1\rangle$ to $|0\rangle$ along a different path $\Gamma_{2}$. The loop $\left[\Gamma_{1}, \Gamma_{2}\right]$ subtends a solid angle $\Omega=2 \phi$ at the center of the Bloch sphere [5] .

Figure 5. The ${ }^{1} H$ and ${ }^{13} C$ spectra obtained for various values of $\chi$, starting from pseudo pure initial state of the spin qubit. The additional low intensity lines in the carbon spectra arise 
from the natural abundant carbon coupled to deuterium in the solvent $\mathrm{CDCl}_{3}$. The sum of $|00\rangle$ and |01) populations, calculated from the normalized line intensities have been plotted as intensities against 37 equidistant values of the phase shift $\chi$ in the range $\left[-360^{\circ}, 360^{\circ}\right]$. (a) $\Omega=0^{\circ}$. No shift in pattern was observed. (b) $\Omega=180^{\circ}$. A shift of $-90^{\circ}$ was observed. The solid line in each plot represents the theoretically expected value $[\mathrm{Eq} 7]$.

Figure 6. The shift in interference pattern as a function of purity of mixed state for $\Omega=$ $60^{\circ}$ (a), $\Omega=90^{\circ}$ (b) and $\Omega=120^{\circ}$ (c). In each plot the shift is plotted for 6 different values of $\mathrm{r}$ ( $\cos \alpha$ ) between 0 and $1 .{ }^{13} C$ and ${ }^{1} H$ spectra are shown for 5 different pairs of $\alpha$ and $\chi$. The solid line in each plot represents the theoretically expected curve according to Eq 18 .

Figure 7. The dependence of interference visibility on mixed state purity for $\Omega=120^{\circ}$ (a), $\Omega=180^{\circ}$ (b) and $\Omega=360^{\circ}$ (c). In each plot the visibility is plotted for 9 different values of $\mathrm{r}(\cos \alpha)$ ranging from 0 to 1 (for $\Omega=180^{\circ}$, $\alpha$ was varied from $0^{\circ}$ to $89^{\circ}$ instead of $90^{\circ}$ in order to avoid the singularity of $\mathrm{Eq}(18) .{ }^{13} \mathrm{C}$ and ${ }^{1} \mathrm{H}$ spectra are shown for 4 different values of $\alpha$. The solid line in each plot represents the theoretically expected curve according to Eq19. 


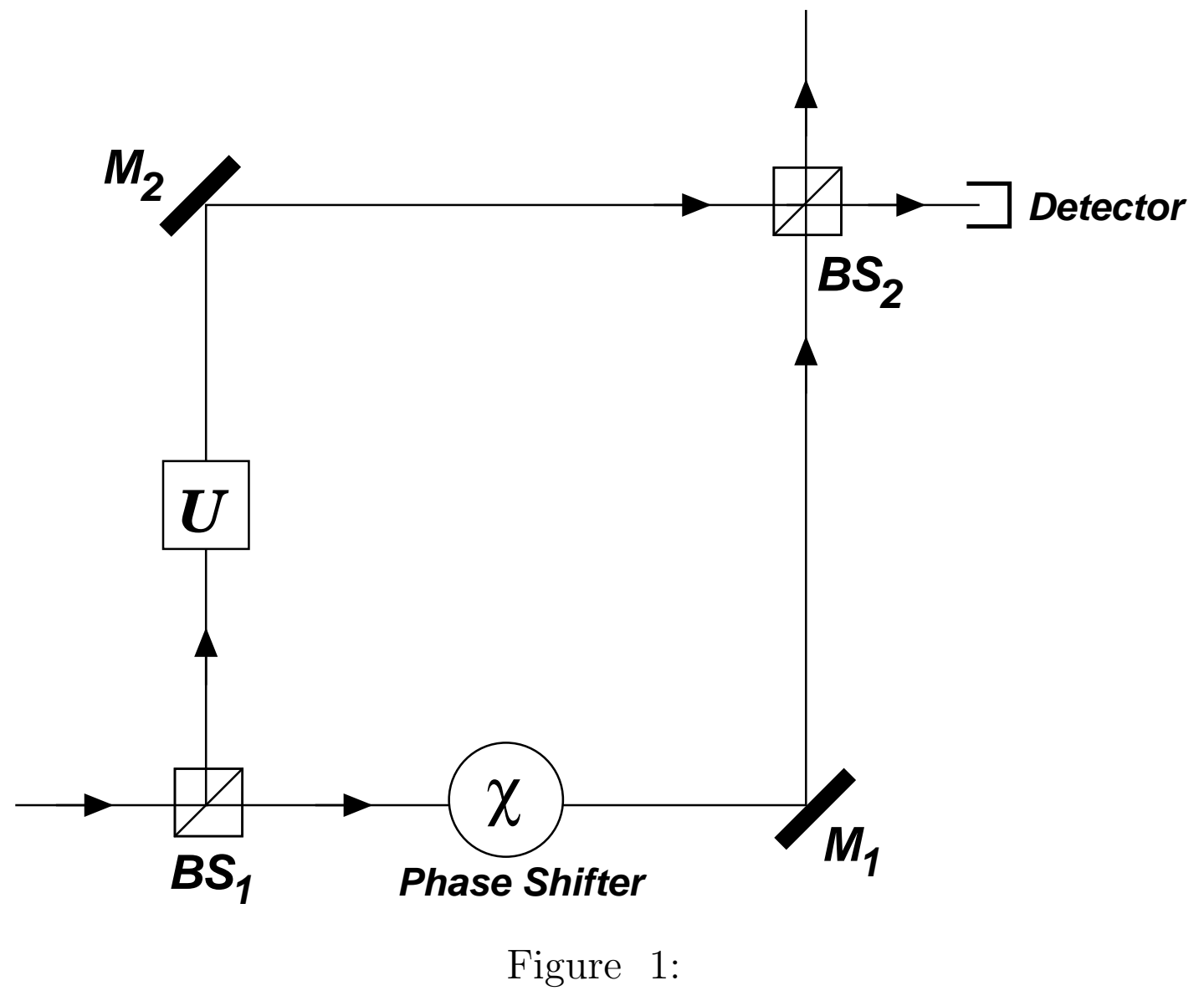




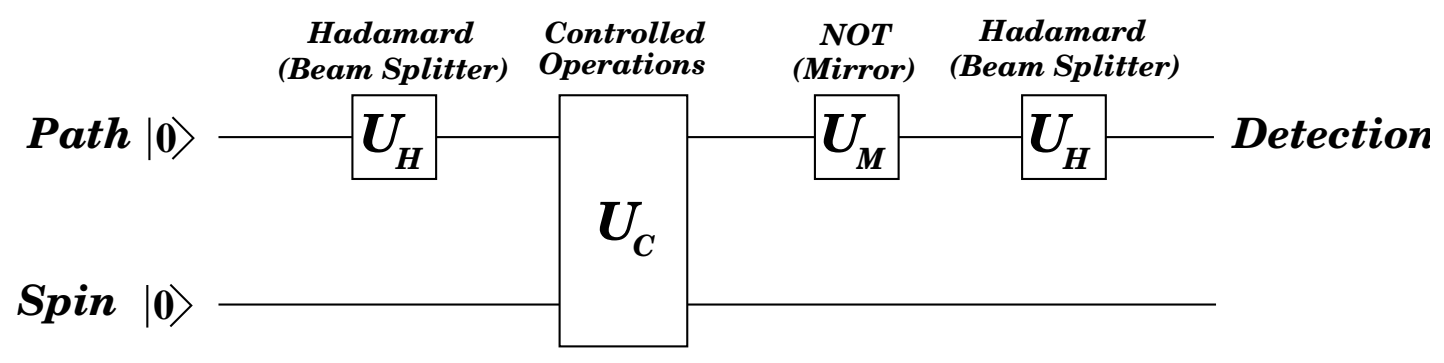

Figure 2: 


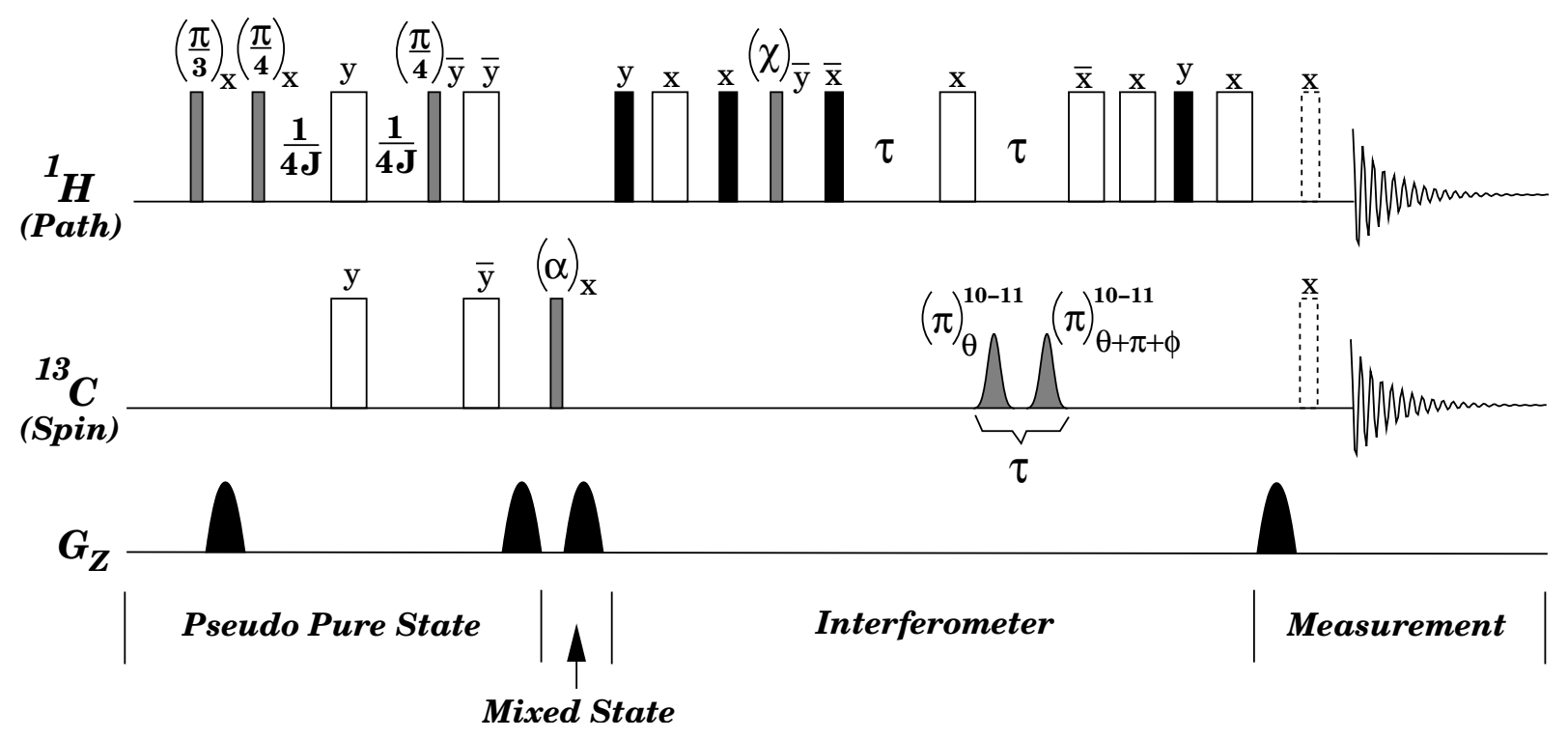

16

Figure 3: 


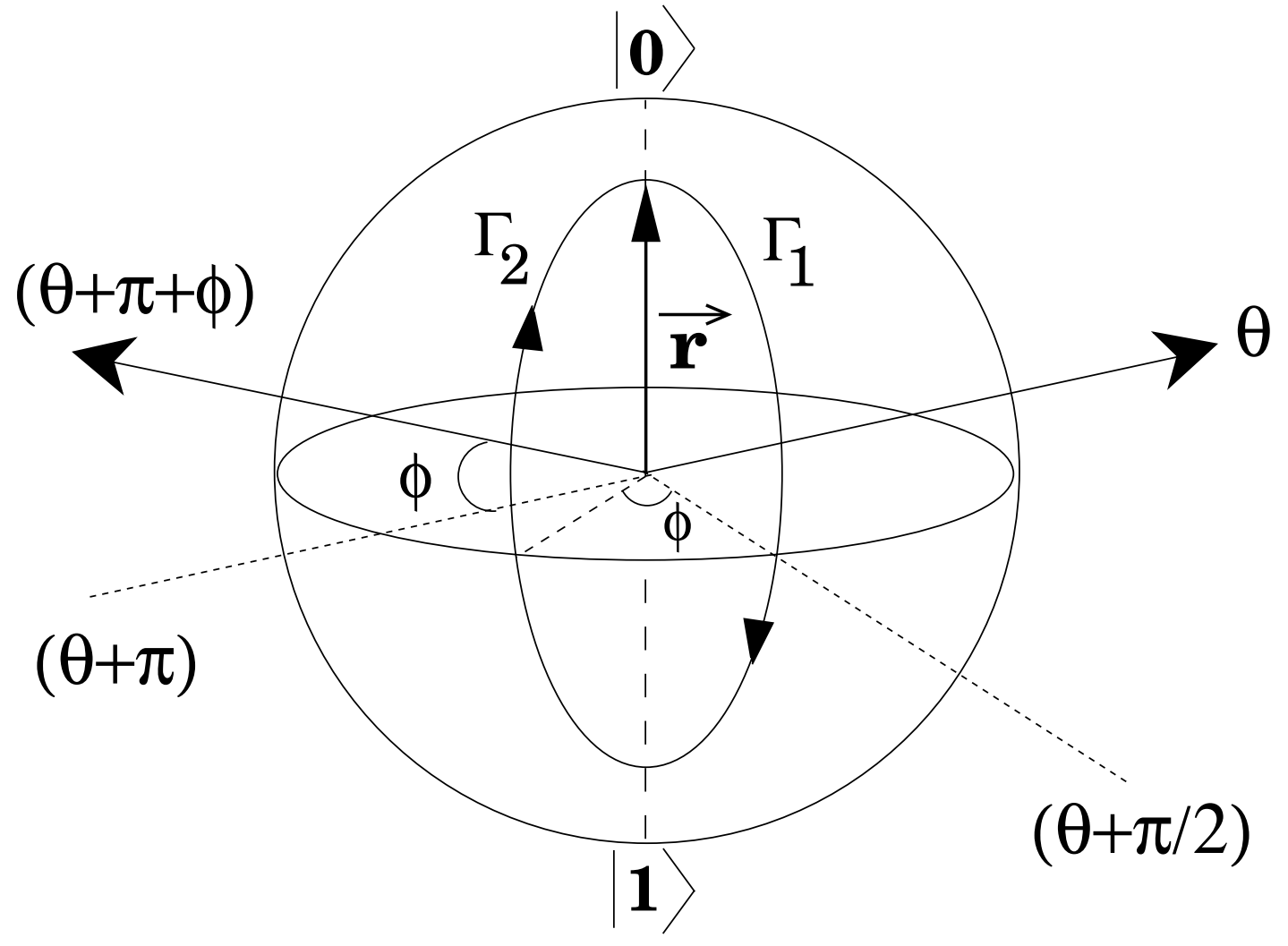

17

Figure 4: 
a)
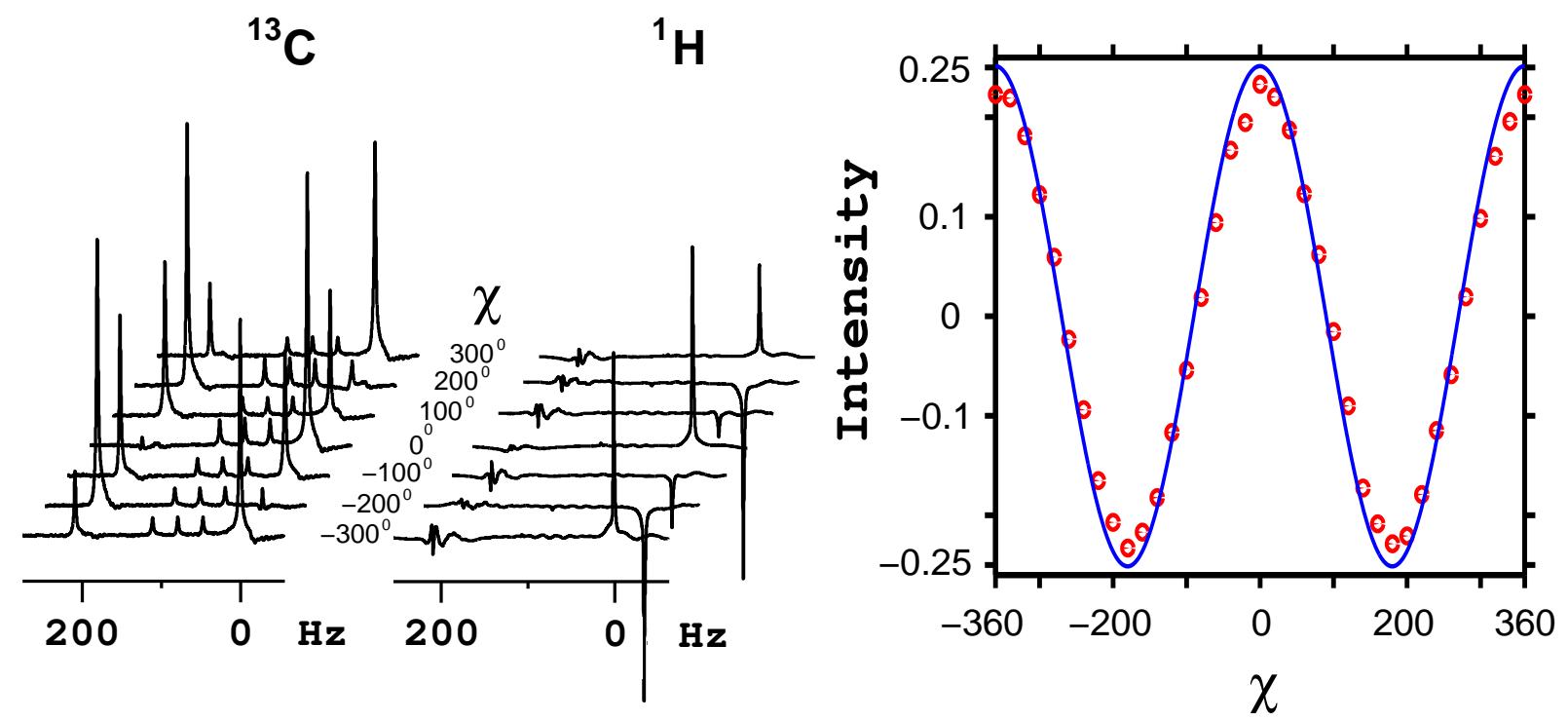

b)
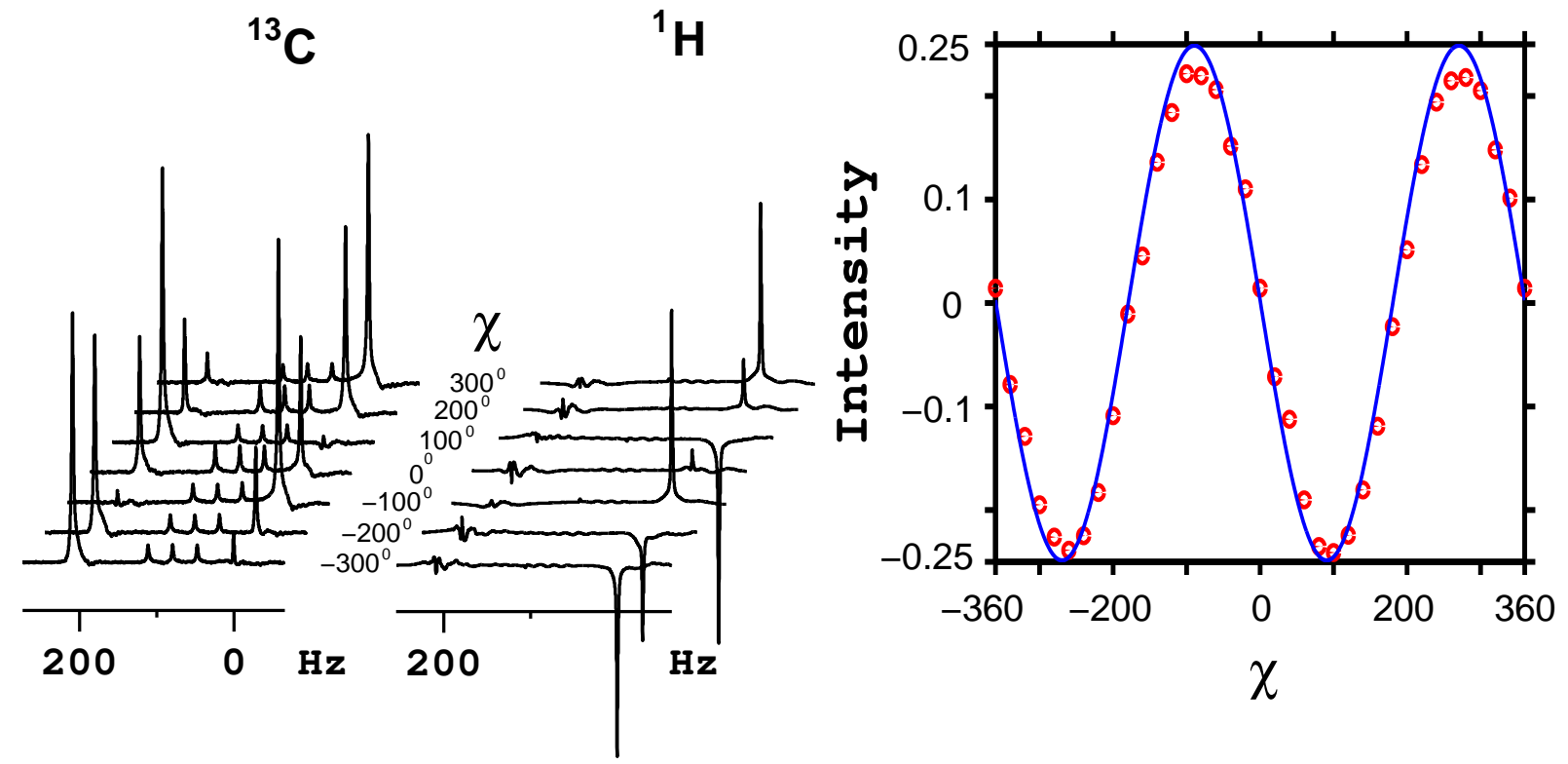

Figure 5: 

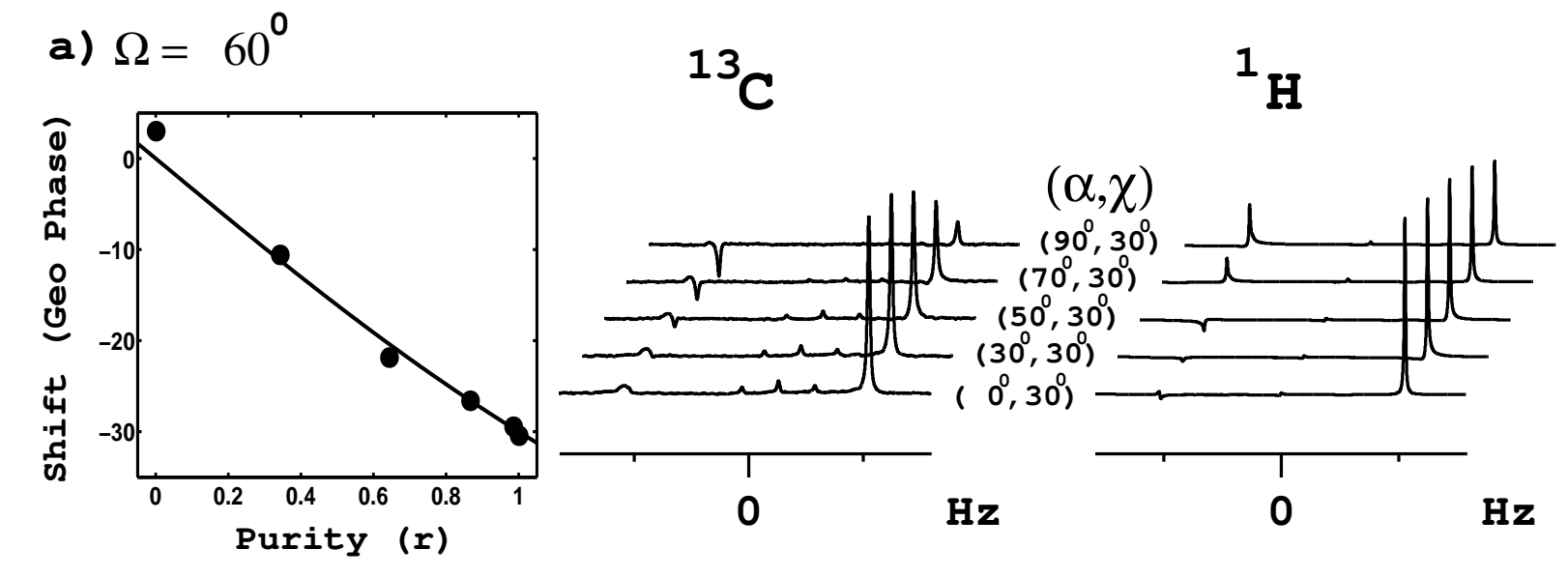

19

b) $\Omega=90^{\circ}$
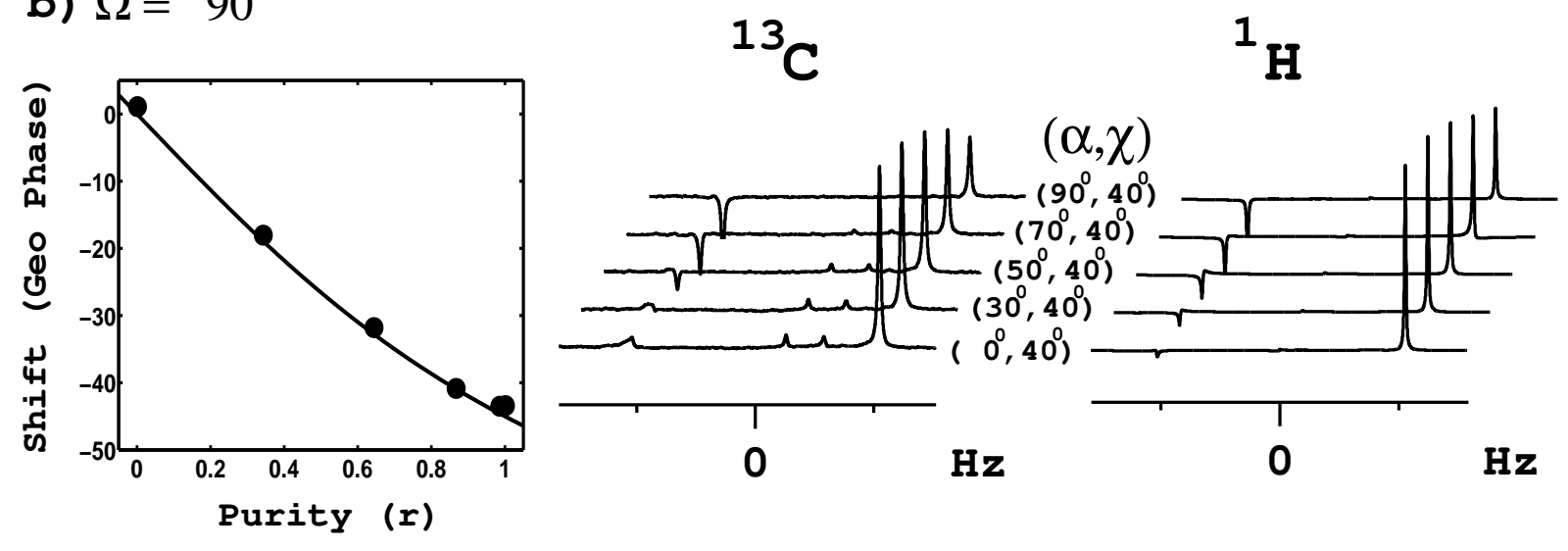

c) $\Omega=120^{\circ}$
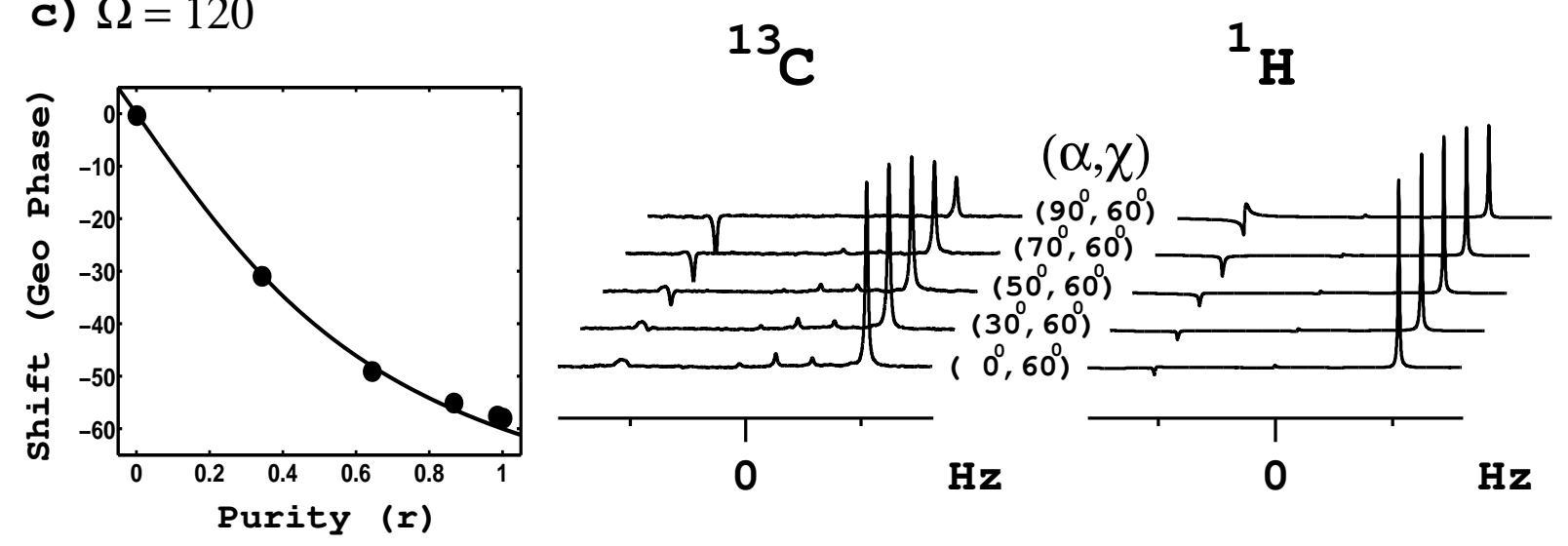

Figure 6: 


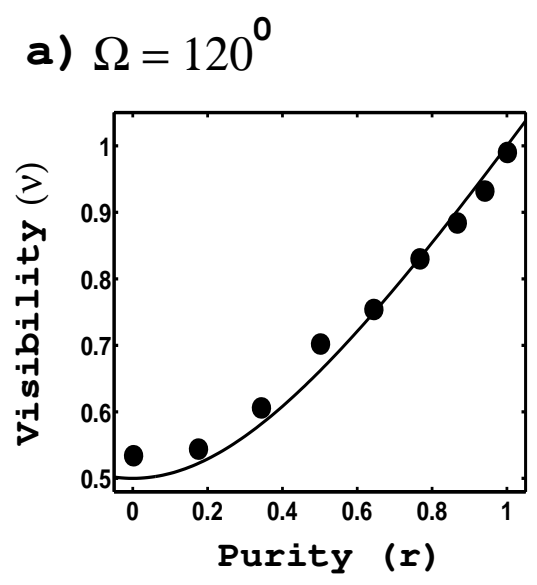

${ }^{13} \mathrm{C}$

${ }^{1} \mathrm{H}$

b) $\Omega=180^{\circ}$

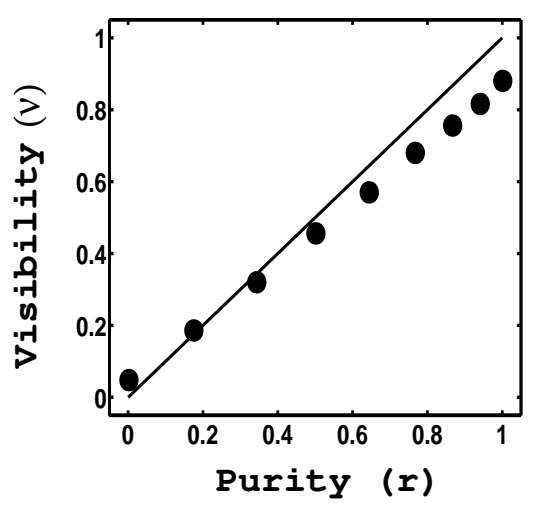

${ }^{13} \mathrm{C}$

${ }^{1} \mathrm{H}$

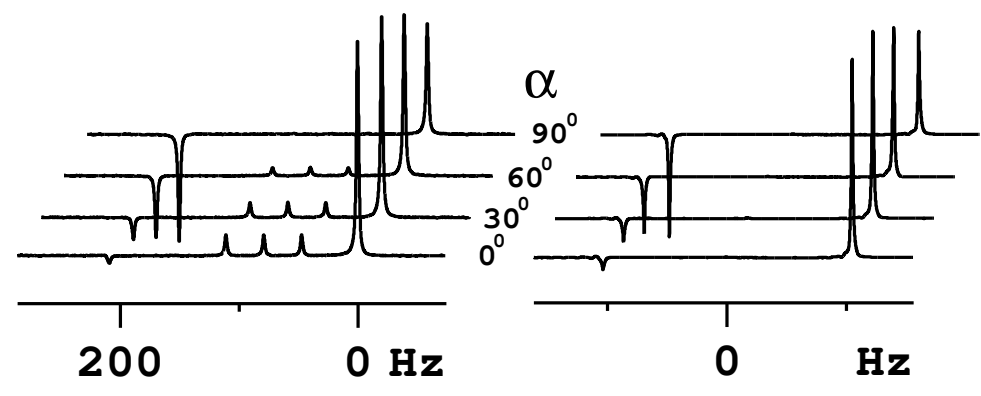

c) $\Omega=360^{\circ}$
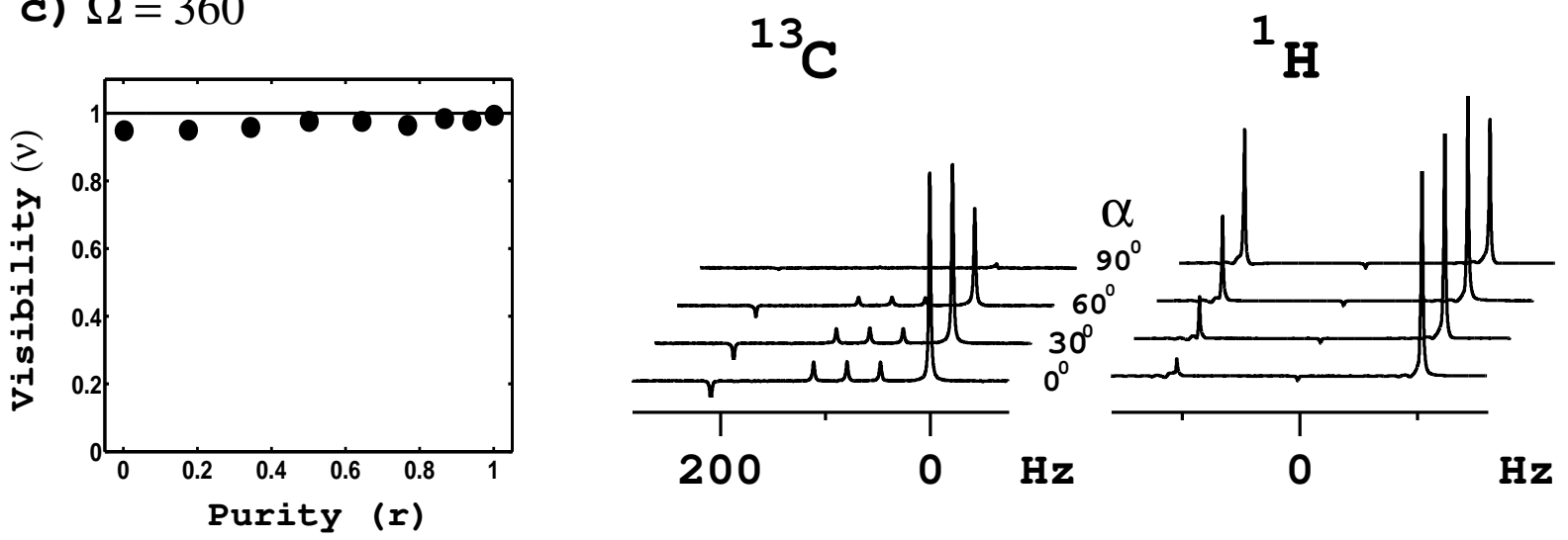

Figure 7: 